MIDPI

MOL2NET, International Conference Series on Multidisciplinary Sciences http://sciforum.net/conference/mol2net-03

sciforum

\title{
QSAR of natural sesquiterpene lactones as inhibitors of Myb-dependent gene expression
}

Gloria Castellano $^{\mathrm{a}, *}$, Lucía Redondo ${ }^{\mathrm{a}}$, Francisco Torrens ${ }^{\mathrm{b}}$

${ }^{\text {a }}$ Departamento de Ciencias Experimentales y Matemáticas, Facultad de Veterinaria y Ciencias Experimentales, Universidad Católica de Valencia San Vicente Mártir, Guillem de Castro-94, E-46001 València, Spain

${ }^{\mathrm{b}}$ Institut Universitari de Ciència Molecular, Universitat de València, Edifici d'Instituts de Paterna, P. O. Box 22085, E-46071 València, Spain

* Corresponding author. Tel.: +34 963544 431; fax: +34 963543274 .

E-mail address: gloria.castellano@ucv.es (G. Castellano)

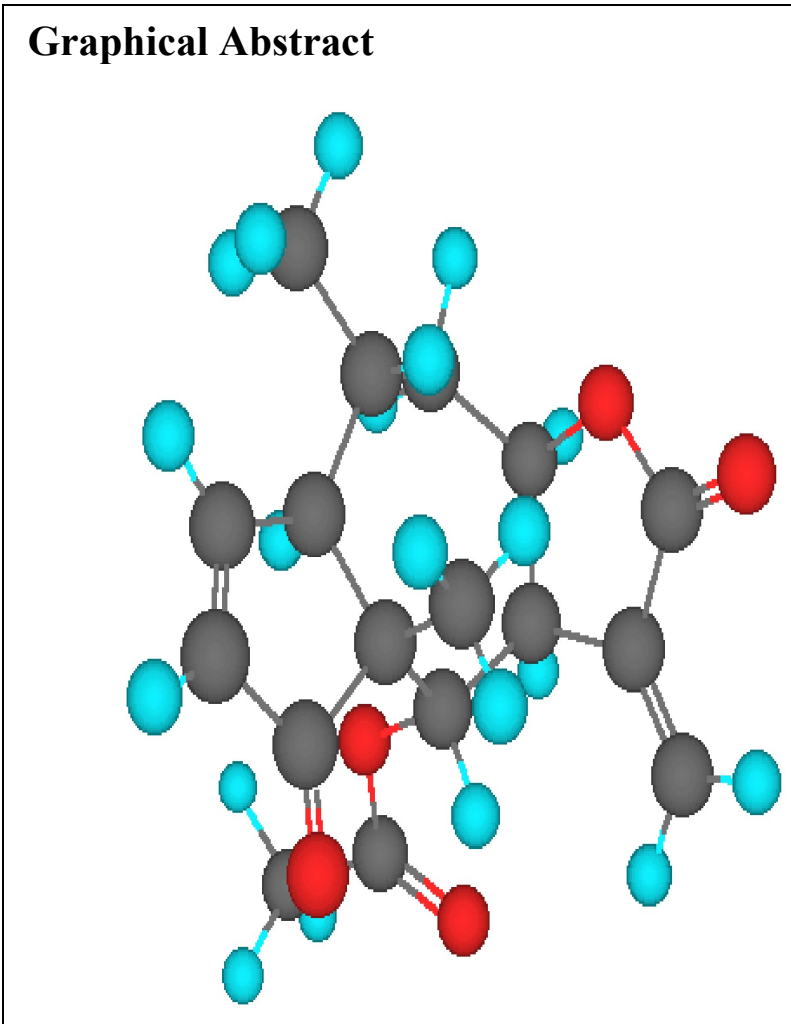

Abstract. Protein c-Myb is a therapeutic target. Some sesquiterpene lactones suppress Myb-dependent gene expression, which results in a mechanism for their potential anti-cancer activity. Database ChEMBL is representative of lactones for physicochemical and physiochemical properties. We studied a dataset with 31 natural lactones are discussed in terms of quantitative structure-activity relationships, which objective is to predict inhibitors of Myb-induced gene expression. Several constitutional descriptors are related to structure-activity. Coefficients standard errors result acceptable in almost all equations. After cross-validation, linear equations for lactones, pseudoguaianolides and germacranolides are the most predictive. Most descriptors are constitutional variables. 